When male Ae. albopictus from the HC population mated with females with the native double infection, all the resulting embryos died, as would be predicted because the females were not infected with the $w$ Pip strain that infected the males (Fig. 1a). However, such embryo lethality did not occur when $w$ Pip-infected males mated with females that were also infected with the $w$ Pip strain (Fig. 1b). Thus, a risk in the authors' approach was that, if any $w$ Pip-infected females were released along with males, they would spread the $w$ Pip infection rapidly through the wild population, eliminating the population-suppressing effects of the $w$ Pip-infected males. This risk was tempered by the finding that $\mathrm{HC}$ females were less susceptible than wild females to infection by dengue and Zika viruses. Therefore, although the goal was population suppression, if $\mathrm{HC}$ females were accidentally released, the worst-case scenario would have been population replacement (Fig. 1b) - still a net gain for public health.

Zheng and co-workers' major innovation was their method of preparing $\mathrm{HC}$ mosquitoes for release. In facilities that mass-rear mosquitoes, male pupae are usually mechanically separated from female pupae on the basis of size differences. Using this procedure to prepare groups of male mosquitoes led to a female contamination rate of approximately $0.2-0.5 \%$, necessitating a secondary, manual screening to remove female pupae, recognized by their distinctive anatomy. However, this labourintensive manual screen substantially limited the total number of mosquitoes that could be prepared. Zheng et al. eliminated the need for the manual screen by subjecting the HC pupae to low-dose radiation that sterilized females but that only slightly impaired male mating success. As a result of eliminating the manual screen, they were able to increase the number of male mosquitoes that could be released by more than tenfold.

Population-suppression strategies crucially depend on the ratio of released males to wild males. Thus, Zheng et al. used mathematical modelling and cage experiments to calculate the optimal sizes and timings of mosquito releases. During the peak breeding season, the rearing facility produced more than 5 million male mosquitoes per week, leading to the release of more than 160,000 mosquitoes per hectare per week at the test sites. Zheng et al. monitored the numbers and viability of eggs produced by wild mosquitoes, as well as the abundance of adult mosquitoes and the rates at which they bit humans at test sites and at nearby control sites (where no HC males had been released).

The releases produced striking results in two successive years. Relative to control sites, the average number of viable eggs produced by wild mosquitoes at test sites declined by $94 \%$ in both years, and the number of wild adult females collected in traps at the two test sites declined by $83 \%$ and $94 \%$ (only female mosquitoes take blood meals). Notably, the estimated human-biting rate decreased by as much as $96.6 \%$. Surveyed support for the releases in the local communities, where residents were initially sceptical or indifferent to the trial, increased from $13 \%$ to $54 \%$.

That Zheng and colleagues' trial almost eliminated a notoriously difficult-to-control vector mosquito from the test sites is remarkable. However, questions remain about the long-term sustainability of their approach. For example, immigrating mosquitoes would inevitably re-establish the natural population once the releases stop. Such recolonization might be prevented by the targeted release of a modest number of males or by conventional vector-control methods, but the required intensity and cost of these additional efforts are unknown. Also unknown is the extent to which this approach can be scaled up spatially. Efforts to develop automated release technologies and more efficient sex-separation methods (for example, see ref. 7), should substantially improve production and release capacity. However, whether such technological advances can overcome the financial and logistical challenges of implementing these approaches at a scale that reduces disease transmission across a major metropolitan area or nationwide remains to be seen.

No single vector-control strategy is expected to fully control populations of disease-carrying mosquitoes; combinations of approaches will probably be most effective ${ }^{8}$. Nevertheless, Zheng and colleagues' work represents a substantial advance, and demonstrates the potential of a potent new tool in the fight against mosquito-borne infectious disease.

Peter A. Armbruster is in the Department

of Biology, Georgetown University,

Washington DC 20057, USA.

e-mail:paa9@georgetown.edu

1. Weaver, S. C., Charlier, C., Vasilakis, N. \& Lecuit, M. Ann. Rev. Med. 65, 395-408 (2018).

2. Bonizzoni, M., Gasperi, G., Chen, X. G. \& James, A. A. Trends Parasitol. 29, 460-468 (2013).

3. Zheng, X. et al. Nature 572, 56-61 (2019).

4. Flores, H. A. \& O'Neill, S. L. Nature Rev. Microbiol. 16 508-518 (2018).

5. Lounibos, L. P. Ann. Rev. Entomol. 47, 233-266 (2012).

6. Armbruster, P. et al. J. Med. Entomol. 40, 356-360 (2003).

7. Gilbert, J. A. \& Melton, L. Nature Biotechnol. 36, 781-782 (2018).

8. World Health Organization. Global Vector Control Response 2017-2030 (WHO, 2017).

This article was published online on 17 July 2019.

\title{
Spectroscopy with a magic twist
}

When two graphene sheets are stacked so that there is a specific angle between them,
fascinating properties reminiscent of high-temperature superconductors emerge.
Spectroscopy now provides insights into this behaviour. SEE LETTERS P.95 \& P.101

\section{MATHIAS S. SCHEURER}

$\mathrm{R}$ otating two overlapping mesh grids relative to each other produces interference patterns called moiré fringes. In the past few years, scientists have started to engineer moiré fringes at the atomic scale by twisting single-atom-thick layers of suitable materials, such as graphene (a 2D honeycomb lattice of carbon atoms). In 2018, it was shown that, as the twist angle between two graphene sheets is tuned to about $1^{\circ}$, the physical properties of the system change dramatically ${ }^{1,2}$ to resemble those of high-temperature superconductors. To explore the microscopic physics of these surprising observations, four teams - Kerelsky et al. ${ }^{3}$ (reporting on page 95), Xie et al. ${ }^{4}$ (page 101), Jiang et al. ${ }^{5}$ in a paper online in Nature and Choi et al. ${ }^{6}$ in a paper on the arXiv preprint server - have carried out spectroscopic measurements on twisted bilayer graphene.

Many properties of single-layer graphene can be qualitatively understood in the free-electron picture, in which the repulsion between electrons is neglected. For instance, the relationship between the energy and the momentum of an electron in this material is, to a good approximation, independent of the density of surrounding electrons.

The situation is very different for twisted bilayer graphene at 'magic' twist angles ${ }^{7}$, the largest of which is about $1^{\circ}$. In this case, electrons occupy flat bands - energy levels whose energies vary only weakly with electron momentum. Because of the small energy range of these flat bands, interactions between electrons cease to be weak perturbations, and the physical properties of the system depend crucially on the electron density. The interactions even induce phases that are not seen in single-layer graphene ${ }^{1,2}$ : the system acts as an electrical insulator for certain electron densities for which the free-electron picture predicts a metallic phase; and, as in the case of high-temperature superconductors, increasing 

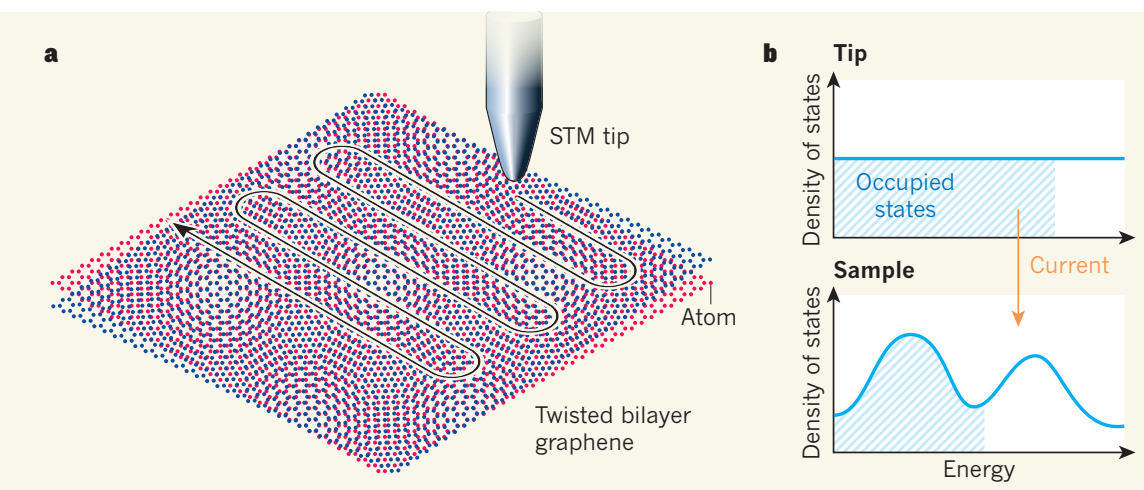

Figure 1 | Spectroscopy of twisted bilayer graphene. a, Four papers ${ }^{3-6}$ report on scanning tunnelling microscopy (STM) experiments in which a conducting tip is scanned across a sample of twisted bilayer graphene. This material consists of two sheets of carbon atoms that are stacked with their honeycomb lattices twisted out of alignment. $\mathbf{b}$, In both the tip and the sample, electronic states are occupied up to a particular energy value. Under an applied voltage (not shown), these values are shifted with respect to each other, causing electrons to 'tunnel' between the tip and the sample. This tunnelling produces a measurable electric current. To a good approximation, the number of states in the tip at a given energy its density of states - is independent of the energy. In that case, the change in the current as the voltage is varied is proportional to the density of states of the sample.

or decreasing the electron density suppresses the insulating behaviour and gives rise to a superconducting phase, in which electrons are transported with zero resistance.

These observations, which are based on measurements of electrical conductivity ${ }^{1,2}$, clearly establish the existence of interactioninduced insulating and superconducting phases. However, the microscopic nature of these phases has remained unknown, and complementary experimental approaches to address this issue are urgently needed. Such an approach is provided by the four current papers, in which the atomic-scale structure and distribution of electron energies are measured.

The groups achieved this measurement using scanning tunnelling microscopy (STM). In this technique, a sharp, conducting tip is scanned across a sample (Fig. 1a). Depending on whether an applied voltage is positive or negative, electrons 'tunnel' from the tip to the sample, or vice versa. The variation of the resulting electric current with the position of the tip encodes the topography of the sample. And the change in the current as the voltage is varied is a measure of the local density of states of the sample - the number of quantum states that can be occupied by electrons at a given energy (Fig. 1b).

Using this technique, the teams visualized the moiré fringes in twisted bilayer graphene. This allowed them to quantify the amount of strain in the system by looking at the variation in the spacing between fringes along different directions. The approach also revealed the spatial rearrangements of the carbon atoms that result from the coupling of the two graphene sheets. These details are crucial for a precise theoretical understanding of the electronic band structure, which constitutes an essential first step towards accurately modelling the insulating and superconducting phases.

In addition, the groups found that the density of states depends sensitively on how many electrons occupy the flat bands. This observation is a direct manifestation of the importance of correlations between the electrons in the system. In particular, the teams found that the density of states at low energies was suppressed for electron concentrations for which an insulator was previously identified ${ }^{2}$. This finding establishes a direct connection between local electronic properties and electron transport.

There are three key differences in the data from the four groups that are worth highlighting. First, Jiang et al. observed only a single peak in the density of states of completely filled or empty flat bands, whereas the other teams saw two peaks. In the commonly used theoretical models of twisted bilayer graphene, two peaks are expected. The cause of this discrepancy is unknown. One possible explanation is that, close to the magic angle, the density of states is highly sensitive to perturbations that can occur during sample preparation.

Second, among the three groups that obtained two peaks, Xie et al. saw the most dramatic effect of electronic correlations when partially filling the flat bands. Not only did they find that the peak with partially occupied electronic states developed a gap-like feature, as was seen by Kerelsky et al. and Choi et al., but they also discovered that the second peak became greatly distorted. This observation is a clear indication of particularly strong correlations.

Third, enhanced breaking of the bilayer system's rotational symmetry is reported to be most prominent for different electron concentrations in the various studies: for the concentrations associated with the insulator ${ }^{3}$; for concentrations near the charge-neutrality point, in which half of the flat bands are filled ${ }^{6}$; and for any concentration as long as the flat bands are partially occupied ${ }^{5}$. This enhanced symmetry breaking is highly likely to be of electronic origin, because its magnitude depends on the density of electrons in the system. However, it is unclear whether it is just a consequence of a strong susceptibility to electronic rotational symmetry breaking that is made visible by strain, which breaks the symmetry weakly, or whether this symmetry breaking is an intrinsic property that is unrelated to strain.

Taken together, the four papers demonstrate that STM measurements on magic-angle twisted bilayer graphene can provide valuable insights into symmetry breaking and the local effect of electronic correlations. Although the results constrain and provide guidance for possible theoretical models, there are many immediate open questions for future STM studies. What is the origin of the discrepancies between the findings of the different groups? Which of the observed phenomena are intrinsic features of twisted bilayer graphene that are robust to perturbations, and which are more fragile? Carrying out STM experiments at a lower temperature than that used in the current studies could shed light on the properties of the superconducting phase and its relation to the insulator, and clarify similarities to and differences from high-temperature superconductivity. In the longer term, quasiparticle-interference measurements, whereby STM is used to probe electronic interference effects around impurities, might also provide complementary information about the system.

More generally, STM measurements similar to those discussed here could be performed on related twisted multilayer systems, such as twisted double-bilayer graphene, which was studied earlier this year ${ }^{8-10}$. There is good reason to think that this young and fast-paced line of research is full of exciting surprises waiting to be discovered, and that spectroscopic techniques will continue to have a key role in this endeavour. Twisted multilayer systems containing graphene and other related materials have a simple chemistry and highly tunable properties, such as electron density. Therefore, it is very likely that these systems will become versatile testbeds for theories of strongly correlated matter.

Mathias S. Scheurer is in the Department of Physics, Harvard University, Cambridge, Massachusetts 02138, USA.

e-mail:mscheurer@g.harvard.edu

1. Cao, Y. et al. Nature $556,43-50$ (2018)

2. Cao, Y. et al. Nature 556, 80-84 (2018).

3. Kerelsky, A. et al. Nature 572, 95-100 (2019).

4. Xie, Y. et al. Nature 572, 101-105 (2019)

5. Jiang, Y. et al. Nature https://doi.org/10.1038/ s41586-019-1460-4 (2019).

6. Choi, Y. et al. Preprint at https://arxiv.org/ abs/1901.02997 (2019).

7. Bistritzer, R. \& MacDonald, A. H. Proc. Natl Acad. Sci. USA 108, 12233-12237 (2011).

8. Shen, C. et al. Preprint at https://arxiv.org/ abs/1903.06952 (2019).

9. Liu, X. et al. Preprint at https://arxiv.org/ abs/1903.08130 (2019).

10.Cao, Y. et al. Preprint at https://arxiv.org/ abs/1903.08596 (2019). 